\title{
A Process Modelling Approach to the Evaluation of Ship Machinery Configuration Alternatives of LNG Carriers
}

\author{
George G. Dimopoulos*, Iason V. Stefanatos and Nikolaos M.P. Kakalis \\ DNV GL Maritime Research \& Development, Piraeus, Greece \\ E-mail: George.Dimopoulos@dnvgl.com
}

Received 07 December 2015, Revised 30 May 2016, Accepted 03 June 2016

\begin{abstract}
The increasing seaborne transportation of Liquefied Natural Gas (LNG) in the current volatile global market and energy supply environment puts a pressure on LNG vessels to be more efficient, environmentally friendly, and costeffective. Modern LNG carriers feature complex and tightly integrated machinery systems to convert the onboard primary energy sources to useful energy demands for propulsion, electricity and heat. Therefore, process modelling and simulation techniques combined with an integrated systems approach is required for the evaluation of different configuration alternatives of LNG carriers. In this paper, we used our in-house process modelling framework DNVGL COSSMOS to develop a generic model of an LNG carrier integrated machinery system encompassing various propulsion and energy recovery technologies. The resulting system model was then coupled with a generic operational profile description accounting for various operating modes and intended trading routes of the vessel. The integrated LNG carrier machinery process model was subsequently used for the evaluation of different technology alternatives and machinery configurations. Namely, the model was used to size the gas-fuel compression trains; assess the introduction and optimal size of an LNG reliquefaction plant; compare electric and mechanical propulsion technologies; and, assess the introduction of energy recovery technologies such as shaft generators and exhaust gas economizers. The model-based studies resulted in an improved insight of this complex integrated machinery arrangement, revealing important performance trade-offs and interrelations between the vessel's sub-systems. The results revealed high energy savings potential of $5 \%$ to $8 \%$ depending on the energy recovery options implemented, operating profile and trading route. At the same time fuel savings of about $6 \%$ were identified, improving the overall cost-effectiveness of the integrated system.
\end{abstract}

Keywords: Process modelling; Integrated energy system; Marine energy systems; LNG carrier;

\section{Introduction}

Natural gas plays a dominant role in the world energy mix, a fact that has intensified its seaborne transport as Liquefied Natural Gas (LNG) by specialized ships (LNG carriers) [1]. These vessels feature complex machinery arrangements mainly due to the fact that LNG is stored onboard insulated atmospheric cryogenic tanks (at about $163^{\circ} \mathrm{C}$ ), with a fraction of the cargo evaporating during voyage (Boil-Off Gas - BOG) and can potentially be used as fuel during operation. In the current shipping landscape, the efficiency, cost-effectiveness, safety, reliability and environmental footprint of these vessels is important for the viability of the marine LNG transport sector. There are also various machinery concepts and configuration solutions available in the market making the design space of the machinery arrangement for these vessels highly complicated. In addition, the vessels operate in highly varying operating profiles and trading patterns, carrying LNG cargoes of varying characteristics.

The question we are addressing in this work is how to compare alternative machinery and propulsion configurations for LNG carriers to support improved decision making. This is a demonstrated need in LNG carriers new buildings, due to their complexity. For that purpose we employ a process modelling approach using our in-house process modelling framework DNVGL
COSSMOS. The aim is to capture the tight interrelations between the components, sub-systems and processes in an LNG carrier marine energy system, and derive improved design solutions at an integrated system level. In the following, the description of the LNG carrier energy system is given, the process modelling approach and model development is presented and the model application on the evaluation of different technology alternatives and machinery configurations for an LNG carrier is described. Our model-based approach is novel and it offers a multitude of benefits with respect to the analysis of these tightly integrated and complex machinery systems. The subsequent sections aim to describe this.

\section{LNG Carrier Integrated Energy System}

The integrated marine energy system of an LNG carrier is considered. The principal energy demands of such vessels are in the form of propulsion power, electricity to cover the hotel and auxiliary systems needs and heat as low pressure steam for steam driven equipment and various heating needs onboard. The primary fuel onboard is the BOG from cargo evaporation in the LNG tanks. In addition, there is also possibility to use standard marine fuels like heavy fuel and gas oil (HFO and MGO, respectively), which are stored onboard. 
Previous studies and the current industry practice have indicated that BOG is the most efficient and cost-effective solution for onboard use [2-6]. It is noted that whenever BOG from natural evaporation cannot cover the entire energy needs, forced evaporation of LNG is applied. The BOG fuel composition (and LHV) is inherently different from natural BOG composition. In addition, due to the natural evaporation process, caused by heat transfer from LNG tanks during voyage, natural BOG has a time-varying composition during voyage with nitrogen and lighter hydrocarbons evaporating first [3].

The main elements and subsystems required to convert the primary fuels' energy to the onboard demand energy forms are depicted in Figure 1. BOG from the LNG tanks is pre-processed passing through a mist separator and in-line mixer and fed to the electric-driven gas compression trains. Then BOG is distributed to the combustion prime mover engines, either for power generation or propulsion. A BOG management module is used to distribute the gas fuel to the prime movers and redirect surplus BOG either to a gas combustion unit (GCU) or to a reliquefaction plant, if present.

The overall system is used to cover a time-varying demand in propulsion, electricity and heat according to its operational profile. In addition, natural BOG flow is also time-varying depending on voyage conditions and LNG cargo type. The integrated energy system of Figure 1 is tightly coupled featuring multiple interrelations and feedback loops between its major subsystems. Both the gas compression trains and reliquefaction plant (if any) are major parasitic electric power consumers. Demand variations affect both parasitic power and BOG use, which in turn may affect its composition. Therefore, an integrated systems approach coupled with process modelling and simulation is used in this work to design, assess and improve LNG carrier machinery systems. In the following sections we employ a computer process model of the LNG carrier integrated machinery system in order to simultaneously address issues and to answer questions on:

- Main engine technology alternatives and system configurations: Electric vs. mechanical propulsion and reciprocating engine technology (dual fuel 4-stroke or 2-stroke).

- Gas compression technology and design: Type of compressors, number and size taking into account variable BOG composition, redundancy and operability.

- Reliquefaction system: Feasibility, cycle configuration and capacity.

- Waste energy recovery alternatives: Waste heat recovery economisers, shaft generators and power management strategies.

- Performance under time-varying operational profile and modes, various trading routes and LNG cargo characteristics.

\section{LNG Carrier Integrated Energy System Process Model}

\subsection{Modelling Framework and Approach}

Process modelling of the LNG carrier integrated energy systems and technology alternatives has been performed in our in-house process modelling framework DNV GL COSSMOS, an acronym for Complex Ship Systems Modeling and Simulation. A detailed description of the COSSMOS framework, the mathematical modelling approach, capabilities and component models can be found in [7]. COSSMOS consists of a library of reconfigurable generic models of ship machinery components. The models capture the steady-state and dynamic thermofluid/ mechanical/transport phenomena/electrochemical behavior of each component. The component model library is coupled with a graphical flowsheeting environment, in which the user can hierarchically synthesize system models of varying complexity. This process results in large systems of non-linear Partial Differential and Algebraic Equations (PDAEs), subject to initial and boundary conditions. The required numerical and optimization solvers are incorporated to our framework in order to perform a wide range of model-based studies such as steady-state and dynamic simulations, parameter estimation, and non-linear, mixed-integer and dynamic optimization. Our framework is implemented in the gPROMS process modelling environment [8].

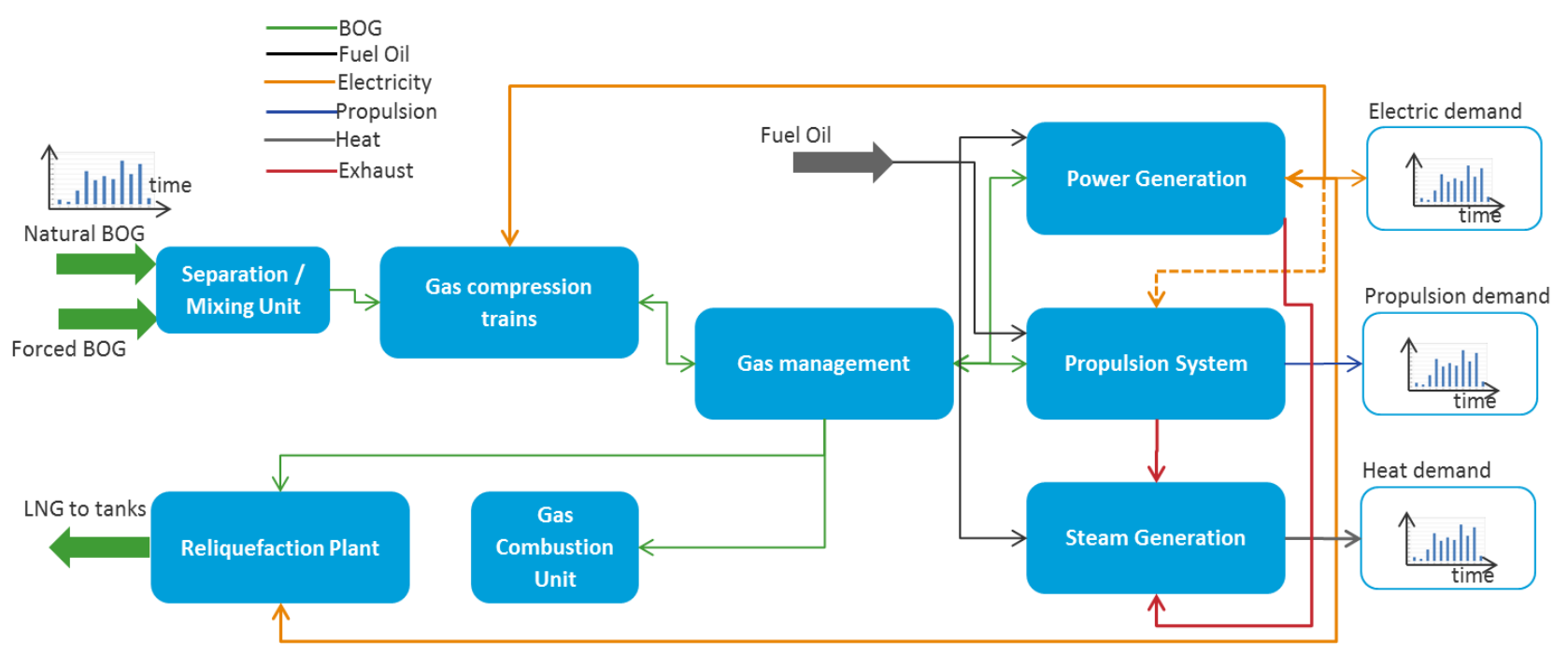

Figure 1. LNG carrier generic marine energy system. 


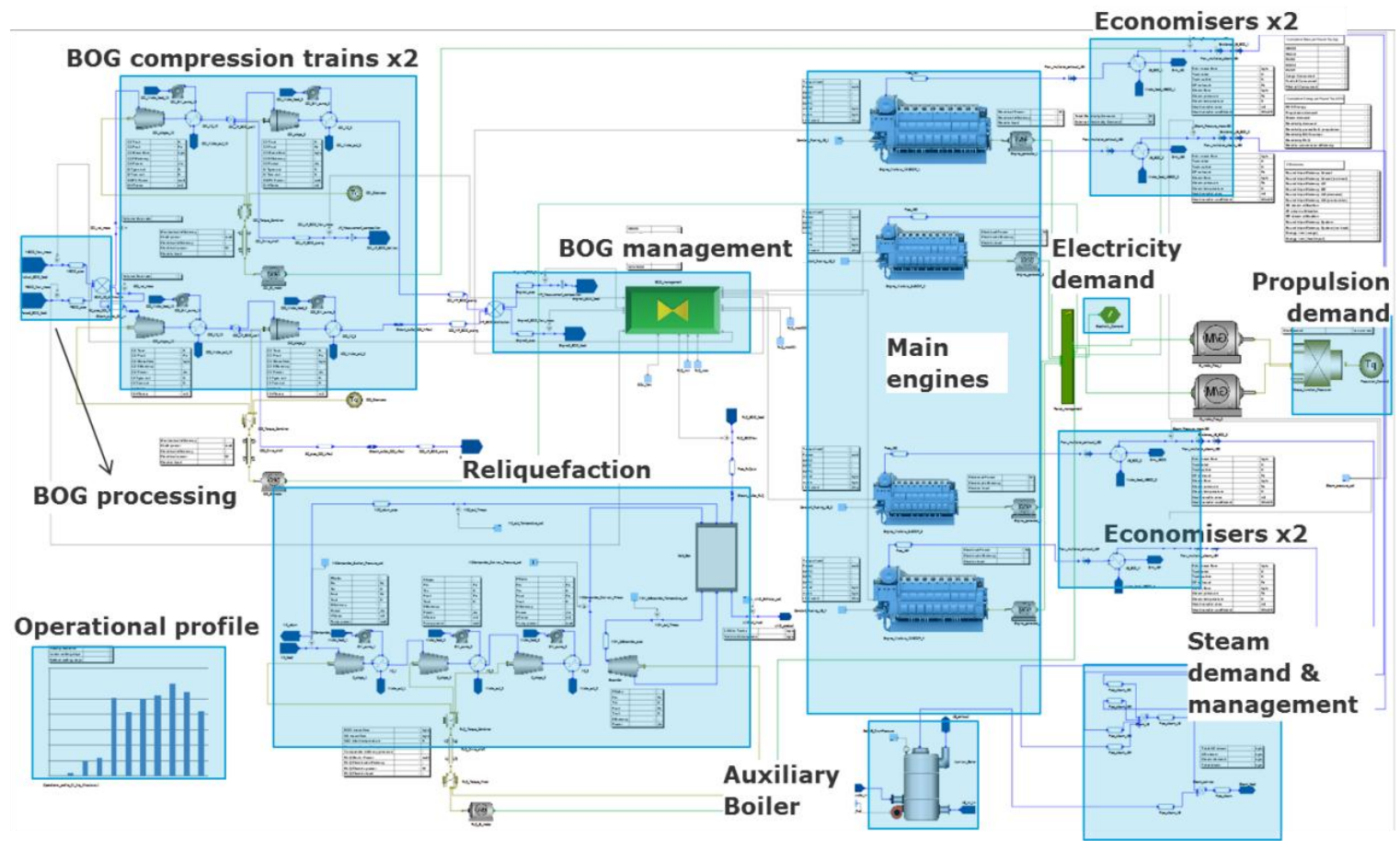

Figure. 2. Generic electric propulsion LNG carrier machinery configuration in the DNV GL COSSMOS framework.

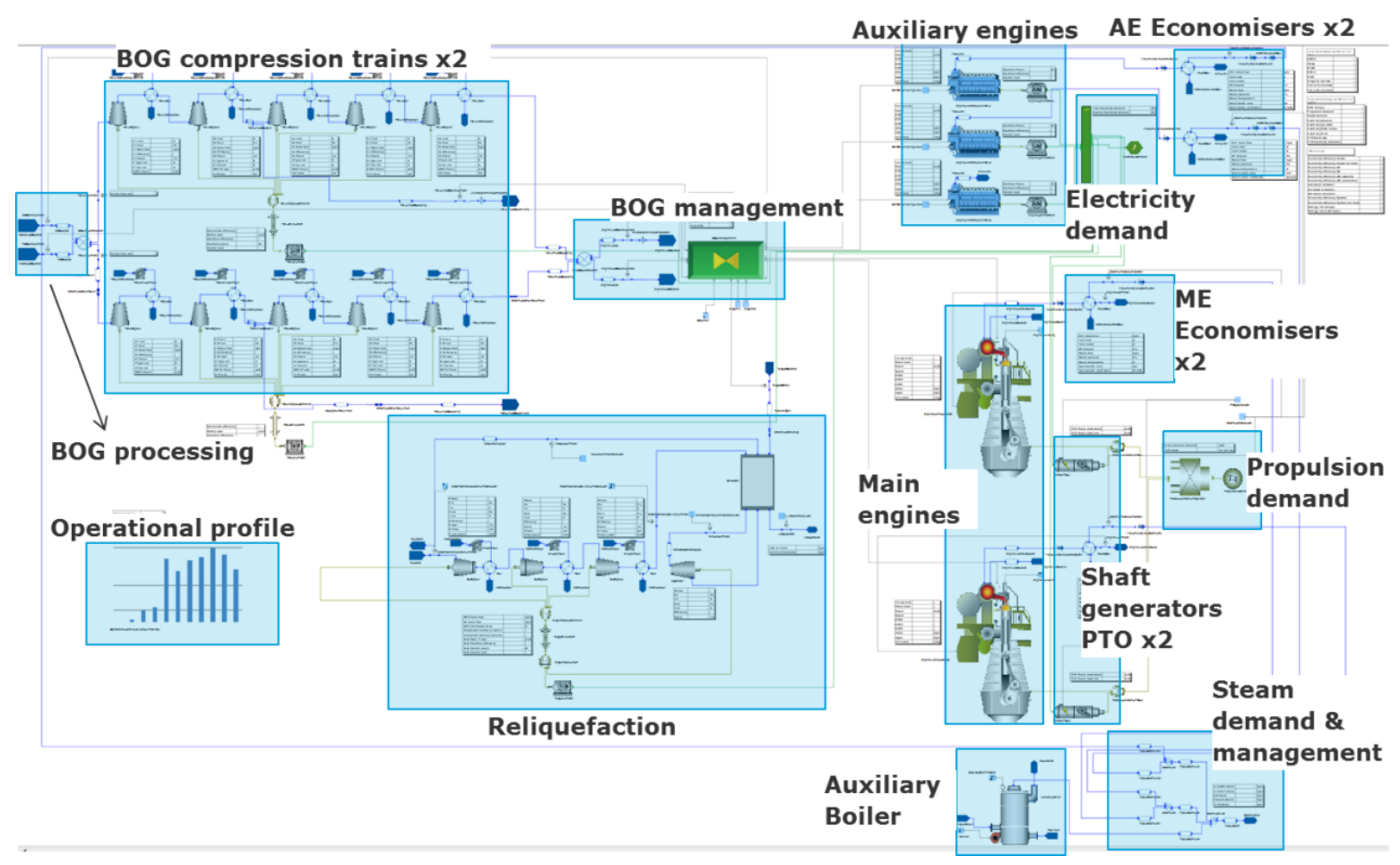

Figure. 3. Generic mechanical propulsion LNG carrier machinery configuration in the DNV GL COSSMOS framework.

Two COSSMOS process models have been developed for electric and mechanical propulsion generic configurations, respectively and are depicted in Figs. 2 and 3. The major component sub-systems featured in these system process models are: the natural and BOG preprocessing module, the BOG compression trains, the BOG management module, the reliquefaction plant, the dual-fuel 4-stroke diesel (DFDE) generator sets (both configurations), the main dual-fuel 2-stroke diesel propulsion engines (mechanical configuration), engine waste heat economizers, auxiliary fired boiler, shaft generators (mechanical configuration), propulsion, electricity and steam demand management modules, and, an operational profile characteristics module. The following paragraphs describe the modelling of each of these major sub-systems. 


\subsection{BOG processing module}

The BOG processing module consists of a natural and forced BOG feed streams, a simplified model of the piping and pressure drop in these sections and a mixing / flash separation component model, Figure 4. In the mixing / separator unit natural and forced BOG streams are mixed and any liquid phase natural gas is removed. All components have been built using standard COSSMOS library elements, as described in [7]. In addition, forced BOG feed composition is equal to that of cargo LNG, which changes according to the trading route and point of loading. Natural BOG composition varies dynamically during voyage as lighter hydrocarbons and nitrogen in the LNG mixture evaporate first. This is captured by the dynamic LNG evaporation model developed in [3].

\subsection{BOG Compression}

BOG compression trains are used to compress the fuel to the required engine operational pressure. There are multiple technologies, arrangements and configurations that can be employed mainly based on electric-driven piston or centrifugal compressors with intercooling, with their number and capacity affected by safety and redundancy issues. For the electric propulsion generic configuration of Figure 2, two-stage centrifugal compressors with intermediate and after cooling are considered. For the mechanical propulsion generic configuration of Figure 3, five-stage piston compressors with intermediate and after cooling are considered. The COSSMOS model of a fivestage, intercooled, electric driven piston compressor is depicted in Figure 5.



Figure. 4. BOG pre-processing sub-system model.
The choice between centrifugal or piston compression technology is mandated by the required gas engine pressure. Four-stroke dual-fuel engines require low fuel pressure ( 6.5 bar), while two-stroke dual-fuel engines require high pressure ( 300 bar). For the latter case piston compressors are the only feasible technology for shipboard applications. In the high pressure case, the possibility for a low pressure extraction from the compression train is also modelled (see Figure 5) in order to feed four-stroke auxiliary engines, as in Figure 3. In both generic system configurations two compression trains are modelled, mainly due to safety and redundancy considerations. The sizing of these trains is part of the subsequent system design analysis.

Compressor stages are modelled using manufacturer performance maps correlating flow, speed, pressure ratio and efficiency. Electric motors drives are modelled as simple electric machines using manufacturer data to derive their efficiency curve. Finally, intercooler heat exchangers are modelled as cross flow plate-fin heat exchangers. It is noted that electric drive power consumption, shaft losses and cooling water pump consumption are accounted for in the compression train model. More information regarding the individual component models can be found in [7].

\subsection{Reliquefaction Plant}

Shipboard reliquefaction plants are usually based on the inverse Brayton cycle with an electric-driven nitrogen cooling loop comprised of multi-stage intercooled compressors and expander [9]. Work produced by the expander is utilized by the compressors to lower the electric drive requirements. This sub-system is also built by using the COSSMOS library of component models [7] and is depicted in Figure 6.

Compressor stages and expander are again modelled using manufacturer performance maps and sea water cooled heat exchanger models for the intercoolers are also used. An essential component model for this sub-system is the cold-box, which is a multi-stream heat exchanger and condenser. In the cold-box BOG is cooled down and condensates to LNG as well as nitrogen is cooled before entry to the expander, both utilizing nitrogen out of the expander as a cooling medium. This component was modelled using the approach followed in $[10,11]$.

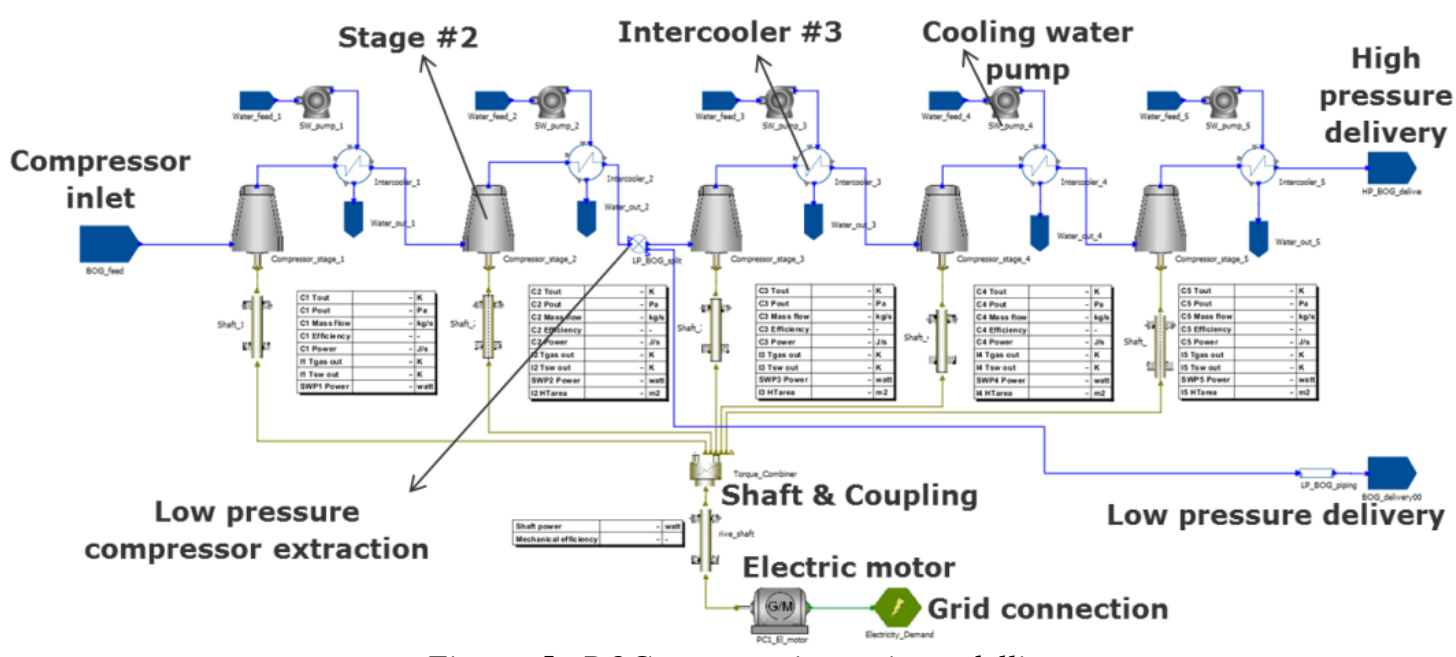

Figure. 5. BOG compression train modelling. 


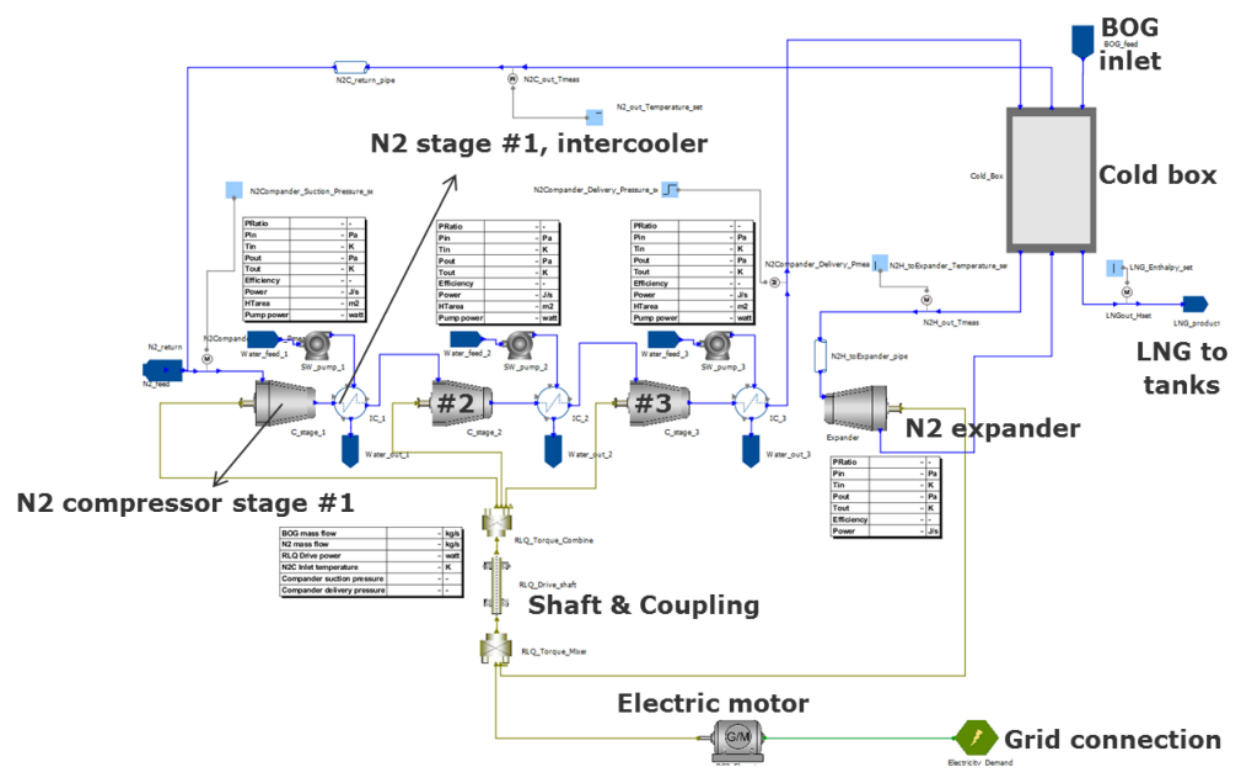

Figure. 6. Reliquefaction plant model.

\subsection{BOG Management}

A BOG management module is used to distribute the gas fuel to the prime movers and redirect surplus BOG either to a gas combustion unit (GCU) or to a reliquefaction plant, if present. It is noted that a GCU is always present due to safety requirements. The goal of this model is to determine the boil-off gas flow to reliquefaction (if present), GCU and forced BOG requirement, according to the available natural BOG and the gas fuel demand from the engines. The following equations hold the determination of excessive and forced BOG quantities:

$$
\begin{aligned}
& \dot{m}_{B O G, \text { exc }}=\max \left\{\dot{m}_{B O G, \text { nat }}-\dot{m}_{B O G, \text { eng },}, 0\right\} \\
& \dot{m}_{B O G, f c d}=\max \left\{\dot{m}_{B O G, \text { eng },}-\dot{m}_{B O G, \text { nat }}, 0\right\}
\end{aligned}
$$

In case of excess BOG and in the presence of a reliquefaction plant flow to GCU is determined:

$$
\dot{m}_{B O G, G C U}= \begin{cases}\dot{m}_{B O G, \text { exc }}-\dot{m}_{R L Q, \text { max }}, & \dot{m}_{B O G, \text { exc }}>\dot{m}_{R L Q, \max } \\ \dot{m}_{B O G, \text { exc }} & , \dot{m}_{B O G, \text { exc }}<\dot{m}_{R L Q, \min }\end{cases}
$$

Equation (3) also entails that the reliquefaction plant can handle BOG flows between its design minimum and maximum operational limits. Finally in the case that no reliquefaction plant is present in the configuration, all of the excess BOG (if any) goes to the GCU.

The consumed LNG at every time instance is:

$$
\dot{m}_{L N G, \text { cons }}=\dot{m}_{B O G, \text { eng }}+\dot{m}_{B O G, G C U}
$$

\subsection{Main and Auxiliary Engines}

In LNG carrier propulsion dual fuel - gas and/or liquid fuel oil - reciprocating engines are a common choice. For power generation (auxiliary electricity or electric propulsion) 4-stroke dual fuel medium speed diesel engines are used. For mechanical propulsion 2-stroke dual fuel slow speed diesel engines are used. Both engine types have two operating modes: gas mode, in which natural gas with a small quantity of pilot fuel oil is used, and, fuel mode, where marine fuel oil is solely used. Both engine types are modelled in COSSMOS using a lookup model based on linear interpolation of catalogued performance data of existing engines as these are given by manufacturers $[12,13]$. The model is presented in $[7,14]$.

\subsection{Steam Generation}

The steam generation elements appearing in the machinery system flowsheets of Figures 2 and 3 are the main and auxiliary engine economizers and a fired auxiliary boiler. These elements are all based on the COSSMOS library generic heat exchanger / evaporator model. Geometry characteristics and semi-empirical heat transfer and pressure drop correlations are used to model the behavior of these components [7]. Engine economizers have a smoke-tube / water drum arrangement, while the auxiliary boiler has a water-tube arrangement. In addition, the auxiliary boiler model is a composite one, featuring also a fuel burner and a steam drum model integrated to the heat exchanger / evaporator model. The steam network also features the main piping elements and pressure drop calculations as well as the mass and energy balance between steam production and demand side that determines the required steam production by the auxiliary boiler, and hence its fuel consumption:

$$
\dot{m}_{s t, D}=\dot{m}_{s t, A B}+\sum_{i} \dot{m}_{s t, E C O, i}
$$

with index $i$ denoting each engine economizer present in the system.

\subsection{Electrical Components \& Power Management}

The electrical component models appearing in the flowsheets are the electric motors, generators, shaft generators, the electric bus, and an electric load element. Electric motors and generators are modelled using a generic motor/generator element of the COSSMOS library utilizing the main specifications of the electrical machine and an efficiency curve over the load range as provided by manufacturer data and commissioning tests [7]. 
The shaft generator model is similar to the previous one with the addition of a maximum power output constraint as a function of the shaft speed, i.e. its operating envelope. The electric bus equates and distributes electricity production with consumers accounting for both active and reactive power. In the case of electric propulsion (Figure 2) the overall power balance of the vessel is:

$$
\sum_{i=1}^{N_{D F D E}} \dot{W}_{e l, i}=\dot{W}_{e l, D}+\sum_{j=1}^{2} \dot{W}_{e l, P M, j}+\dot{W}_{e l, \text { parasitic }}
$$

In the case of mechanical propulsion, with shaft generators installed on both propulsion engines the power balance is:

$$
\sum_{i=1}^{N_{D F D E}} \dot{W}_{e l, i}+\sum_{j=1}^{2} \dot{W}_{e l, S G, j}=\dot{W}_{e l, D}+\dot{W}_{e l, \text { parasitic }}
$$

In both cases the parasitic electricity consumption accounts for the electric power required for the reliquefaction plant, the $\mathrm{BOG}$ compression trains and the various sea water cooling pumps. Finally, according to the total demand at each operating mode, the power management module determines which and how many generating sets operate.

\subsection{System integration}

The basic steps to build the integrated machinery system model in COSSMOS are as follows:

- Selection of configuration alternatives to be examined.

- Data gathering for the individual components and subsystems.

- Data gathering of operational profiles and trade route characteristics.

- Components and sub-system models calibration and validation.

- Complete system model building, including trade and operational profile.

- Derivation of the system-wide techno-economic metrics.

- Simulation campaign and model-based studies..

\section{Application Case}

\subsection{Case Study Vessel}

An LNG carrier vessel with a cargo capacity of 175000

$\mathrm{m}^{3}$ is considered. The main design characteristics of the vessel are given in Table 1. A trading route from USA (east coast) to Japan is considered, with a sailing distance of 9700 nautical miles (nm) and an LNG cargo composition, as given in Table 2 .

In addition, a typical realistic operational profile of the vessel is assumed. The profile has 24 operating modes, accounting for a complete round-trip of the vessel: starting with an empty ship (ballast) anchored waiting, loading cargo, then a loaded voyage broken down to 10 speed segments, anchored loaded, unloading and a ballast voyage also broken down to 10 speed segments. It is noted that the 20 loaded and ballast voyage modes have a variable duration, depending on the sailed distance and vessel speeds distribution. Typical speed segments and their percentage of sailing time have been statistically derived from operational data of similar ocean-going LNG carriers, and are given in Figure 7. The remaining non-sailing modes have fixed durations of 34, 24, 12 and 14 hours for the anchored ballast, loading anchored loaded and unloading modes, respectively.

\begin{tabular}{|c|c|}
\hline Characteristic & Value \\
\hline Cargo capacity & $175000 \mathrm{~m}^{3}$ \\
\hline Length & $290 \mathrm{~m}$ \\
\hline Breadth & $45 \mathrm{~m}$ \\
\hline Depth & $26 \mathrm{~m}$ \\
\hline Design draft & $12 \mathrm{~m}$ \\
\hline Service speed & $19.5 \mathrm{kn}$ \\
\hline Design boil-off rate & $0.15 \%$ / day* \\
\hline Number of propellers & 2 \\
\hline Design propulsion power & $2 \times 12500 \mathrm{~kW}$ \\
\hline $\begin{array}{l}\text { Installed dual fuel diesel } \\
\text { generating sets }^{(\mathrm{E})}\end{array}$ & $\begin{array}{l}2 \times 11400 \mathrm{~kW} \\
2 \times 5700 \mathrm{~kW}\end{array}$ \\
\hline Installed main engines $^{(\mathrm{M})}$ & $2 \times 12600 \mathrm{~kW}$ \\
\hline Installed auxiliary gen-sets ${ }^{(\mathrm{M})}$ & $3 \times 3950 \mathrm{~kW}$ \\
\hline Auxiliary boiler capacity & $6500 \mathrm{~kg} / \mathrm{h}$ \\
\hline Service steam & 7 bar (sat.) \\
\hline
\end{tabular}

Table 1. LNG carrier key design characteristics.

* Corresponds to a value of $0.15 \%$ of design cargo capacity per day, a characteristic of the tank insulation technology.

${ }^{\mathrm{E}}$ Electrical propulsion arrangement, Figure 2.

${ }^{\mathrm{M}}$ Mechanical propulsion arrangements Figure 3.

Table 2. LNG carrier key design characteristics.

\begin{tabular}{lc}
\hline Species & Molar fraction \% \\
\hline Methane & 98.012 \\
Ethane & 1.440 \\
Propane & 0.228 \\
Butane & 0.002 \\
Isobutane & 0.006 \\
Nitrogen & 0.312 \\
\hline
\end{tabular}

Finally, for each one of these 24 operating modes typical expected propulsion power, electric power, steam flow rate, BOG flow rate and vessel speed are also derived. Having the route's sailing distance, the expected speed at each speed segment and the distributions of Figure 7, the hours in each of the sailing modes are determined. These also yield an average number of 5.3 trips per year for the given route. In Figure 8 the aforementioned demand profiles are given in normalized figures with respect to their design values.

\subsection{Performance and efficiency metrics}

A set of performance metrics were introduced in order to facilitate the comparison of the various LNG carrier machinery design alternatives, capturing the effect of: integrated system behavior, influence of trading route, introduction of energy recovery options and multiple fuels used. The instantaneous (per operating mode) vessel overall efficiency is:

$$
\eta_{I N S T, i}=\frac{\dot{W}_{p r, D, i}+\dot{W}_{e l, D, i}+\dot{Q}_{s t, D, i}}{\dot{E}_{L N G, \text { cons }, i}+\dot{E}_{H F O, c o n s, i}+\dot{E}_{P F O, \text { cons }, i}}
$$


and by considering all operating modes, the round-trip vessel overall efficiency is:

$$
\begin{gathered}
\sum_{i=1}^{N_{\text {modes }}}\left(\dot{W}_{p r, D, i} \cdot \Delta \tau_{i}\right)+\sum_{i=1}^{N_{\text {modes }}}\left(\dot{W}_{e l, D, i} \cdot \Delta \tau_{i}\right)+ \\
\sum_{i=1}^{N_{\text {modes }}}\left(\dot{Q}_{s t, D, i} \cdot \Delta \tau_{i}\right) \\
\sum_{i=1}^{N_{\text {modes }}}\left(\dot{E}_{L N G, \text { cons }, i} \cdot \Delta \tau_{i}\right)+\sum_{i=1}^{N_{\text {modes }}}\left(\dot{E}_{P F O, \text { cons }, i} \cdot \Delta \tau_{i}\right)
\end{gathered}
$$$$
\varepsilon_{R N D}=\left(\begin{array}{l}
\sum_{i=1}^{N_{\text {modes }}}\left(\dot{E}_{L N G, \text { cons }, i} \cdot \Delta \tau_{i}\right)+ \\
\sum_{i=1}^{N_{\text {modes }}}\left(\dot{E}_{H F O, \text { cons }, i} \cdot \Delta \tau_{i}\right)+ \\
\sum_{i=1}^{\text {modes }}\left(\dot{E}_{P F O, \text { cons }, i} \cdot \Delta \tau_{i}\right)
\end{array}\right) / 2 \cdot S_{\text {route }}
$$

Finally, the fuel-related expenses per round-trip are defined:

$$
\begin{aligned}
\text { OPEX } & =c_{L N G} \cdot \sum_{i=1}^{N_{\text {modes }}}\left(\dot{m}_{L N G, \text { cons }, i} \cdot \Delta \tau_{i}\right)+ \\
& c_{H F O} \cdot \sum_{i=1}^{N_{\text {modes }}}\left(\dot{m}_{H F O, \text { cons }, i} \cdot \Delta \tau_{i}\right)+ \\
& c_{M D O} \cdot \sum_{i=1}^{N_{\text {modes }}}\left(\dot{m}_{P F O, \text { cons }, i} \cdot \Delta \tau_{i}\right)
\end{aligned}
$$

The OPEX metric is heavily dependent on the energy efficiency metrics but it is not directly analogous to efficiency. This is due to the differences in the price of the three fuels simultaneously used onboard.

\section{Results}

\subsection{Compressor Train Sizing}

The first model-based study, that the generic LNG machinery configuration models were used, addressed the sizing of compression trains for the mechanical propulsion option (Figure 3). HP compression trains are a major cost item of the entire system, subject to safety and redundancy constraints. The compression train sub-system has to be capable of covering the maximum gas fuel flow (worst case). In addition, normal sea-going operation has to be attained with one compressor out of service. Therefore, three options were considered: 2 compressors sized at 100\% of maximum flow each, 2 compressors at $75 \%$ of maximum flow, and 3 at $50 \%$.

Using the system model of Figure 3 for the case study vessel and operational profile of Section 4, the solution of having 2 compressors sized at $75 \%$ of maximum flow each was identified as the most cost-effective. In Figure 9, the simulation results of the actual performance during operation (marked with "x") are compared to the characteristic limits of compressor. The limits denote compressor maximum power over flow for the different combinations of HP (300 bar) delivery and LP (6.5) delivery ratios. The compressor can fulfil its mission when all operational points are below their respective maximum power curve. This problem requires a model-based integrated systems approach since the HP/LP ratio varies considerably during operation (from 0 when ship is not sailing to 4 when ship is sailing at high speeds) and, therefore, it is hard to determine if the operational constraints are satisfied without considering the complete system operation. The simulation results indicate that by selecting the $2 \times 75 \%$ compressors, one unit is sufficient for normal operation. A similar approach was also followed for the sizing of the compressors for the electric propulsion configuration of Figure 2, again $2 \times 75 \%$ of maximum flow.

\subsection{Reliquefaction Plant}

Subsequently, the use of a partial BOG reliquefaction plant was investigated to recover the excess boil-off in all operating modes that fuel energy demand is lower than natural BOG. The techno-economic feasibility of the reliquefaction plant is linked to the amount of excess boiloff during system operation, which is determined by the efficacy of the tank insulation (namely, design boil-off rate $=0.15 \%$ /day, see Table 1) and the overall efficiency of the propulsion system.
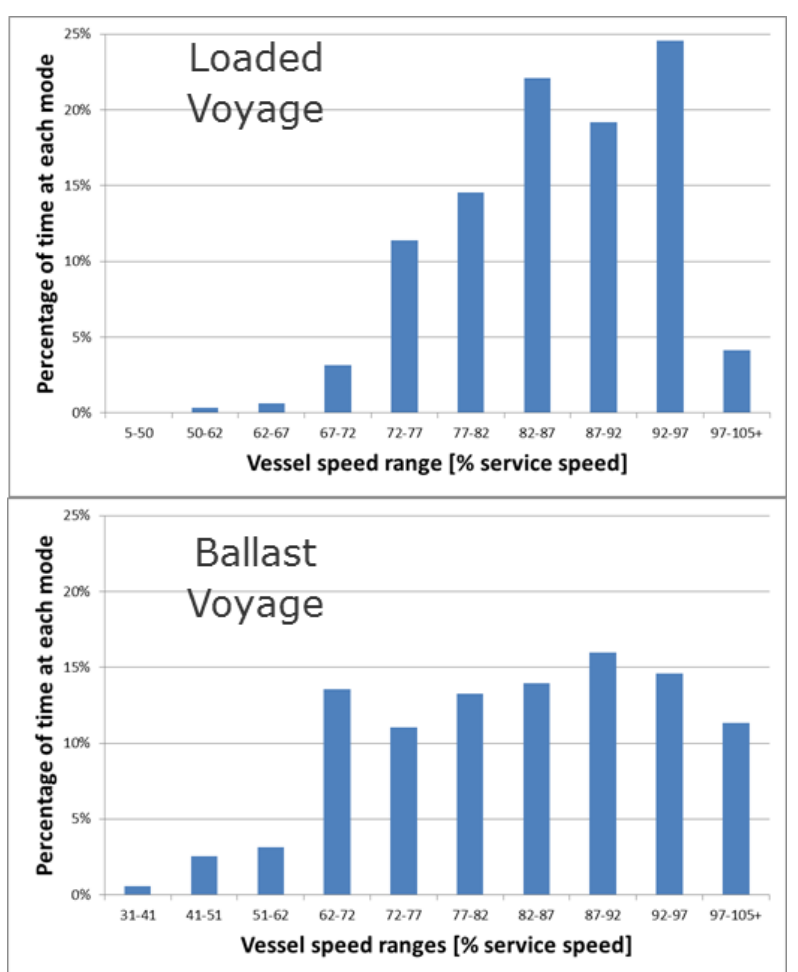

Figure. 7. Sailing speed statistical distributions for loaded and ballast voyages.

A reliquefaction system, as in Figure 6, was investigated for different nominal BOG flow capacities, with the results of this parametric study presented in Figure 10. The results indicate that systems with a capacity greater than $1500 \mathrm{~kg} / \mathrm{h}$ have a marginal improvement on the actual quantity of BOG reliquefied during a round-trip for both configurations. Namely, a system of $2 \mathrm{tn} / \mathrm{h}$ capacity liquefies $2-3 \%$ less BOG than a $2.5 \mathrm{tn} / \mathrm{hr}$ system, which can liquefy $100 \%$ of the excess BOG, Figure 10a. Therefore, a $20 \%$ reduction in size (and capital cost) has a relatively small effect in the efficiency and utilization of the system. The electric power / energy required to drive the systems is shown in Figure $10 \mathrm{~b}$ as a fraction of the electricity demand. These results indicate that the reliquefaction plant's operation is a major parasitic electric 


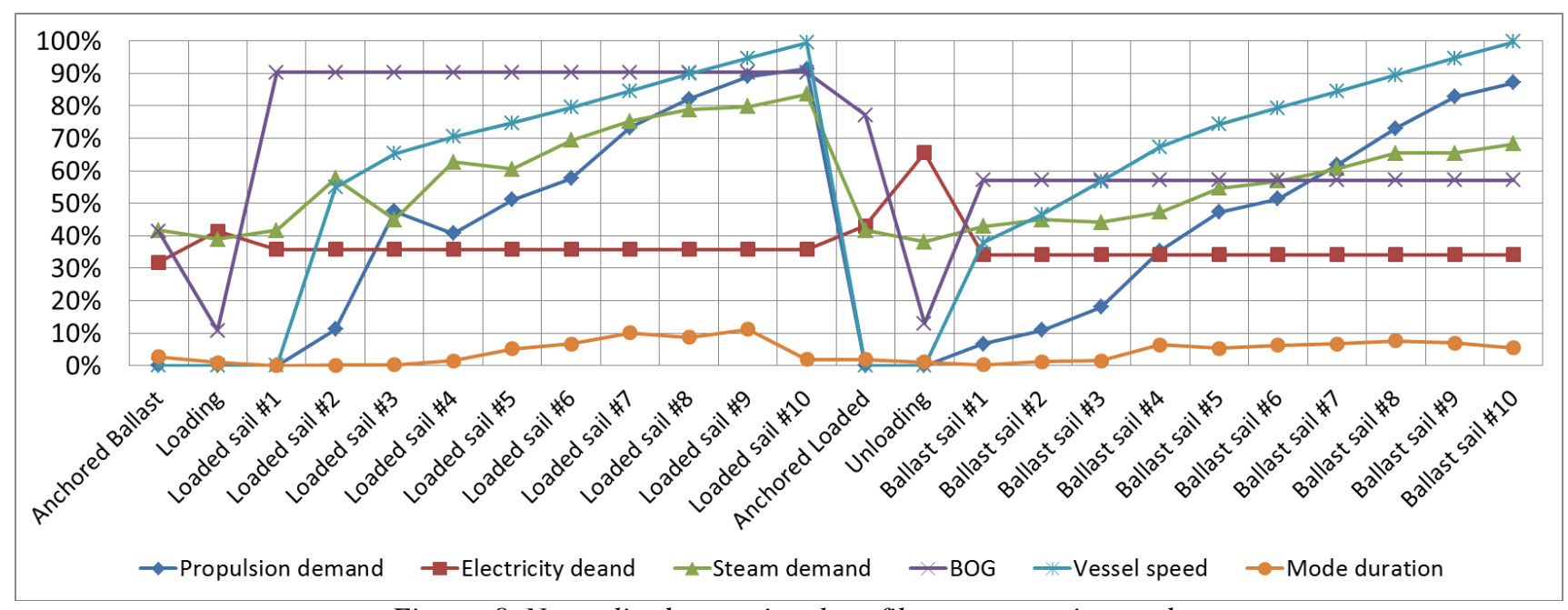

Figure. 8. Normalized operational profiles per operating modes.



load. Approximately, $15 \%$ of additional BOG fuel, compared to the liquefied BOG quantity, is required for reliquefaction plant operation. In the mechanical configuration, larger BOG quantities are reliquefied: approximately $60 \%$ more due to the fact that the mechanical configuration has a better overall efficiency than the electrical propulsion one (see paragraph 5.3); at the expense, however, of increased electricity consumptionFinally, the payback periods of the various system capacities for both electric and mechanical propulsion configurations are shown in Figure 10c. These payback periods were calculated based on the additional cargo revenue of the reliquefied BOG. For the subsequent configurations analysis a reliquefaction system of 2000 $\mathrm{kg} / \mathrm{h}$ capacity was selected for both the electric and mechanical configurations. This system has a payback period of 4 and 6 years for the mechanical and electric configuration, respectively.

\subsection{Alternative configurations \& energy recovery options}

The aim of this study was to assess the techno-economic performance of the electric and mechanical propulsion configurations (Figures 2 and 3) for the case study vessel, trading route and operating profile (Section 4). In addition, the following energy recovery variants for the mechanical propulsion configuration (Figure 3) were simulated:

- Auxiliary engine economisers (AEECO) with rated capacity: 2 x $1000 \mathrm{~kg} / \mathrm{hr}$.

- Shaft generators (PTO) with rated power output: $2 \mathrm{x}$ $1000 \mathrm{~kW}$

- Combined AEECO and PTO installation.

It is also noted that in the electric propulsion configurations all engines were assumed to be equipped with economizers and in the mechanical propulsion configuration main engines also featured exhaust gas economizers (MEECO). Finally, as the results of paragraph 
5.2 indicated, a reliquefaction plant of $2000 \mathrm{~kg} / \mathrm{h}$ capacity is installed in both configurations.

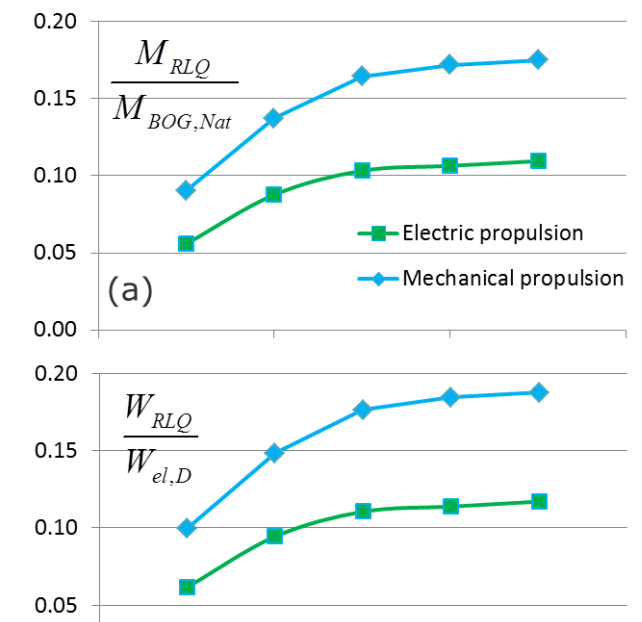

(b)

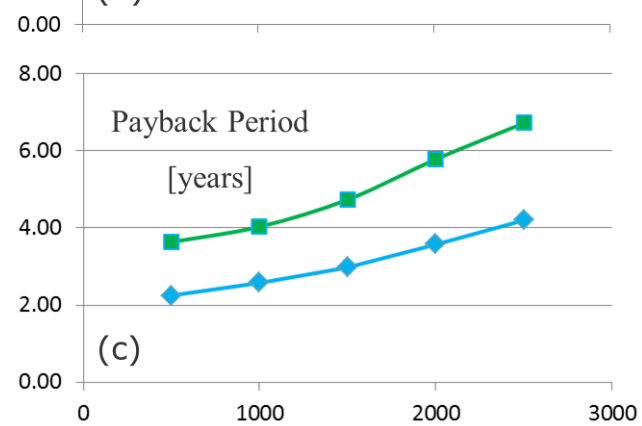

Reliquefaction system nominal BOG capacity $[\mathrm{kg} / \mathrm{h}]$

Figure. 10. Reliquefaction system appraisal for the electric and mechanical propulsion configurations.
In Figure 11, the round-trip vessel overall efficiency, specific energy consumption and fuel operating expenses are given. In Table 3, steam and electricity demand coverage ratios for the different energy recovery variants for both configurations are given. It is noted that the specific energy consumption and fuel operating expenses are given in non-dimensional form, divided by the respective values of electric propulsion configuration results (baseline).

The results indicate that the mechanical configuration yields a $4.5 \%$ improvement in overall system efficiency with a corresponding $3.2 \%$ reduction in fuel OPEX, compared to the electric propulsion one. This roughly corresponds to 0.5 million USD annual savings. In the electric propulsion configuration the auxiliary boiler usage is minimized (Table 3) since the economizers of the 4stroke dual fuel engines can cover the entire steam demand due to their high exhaust heat content. On the other hand 2stroke dual fuel engines, in the mechanical configuration, have considerably lower exhaust heat content, resulting in significant utilization of the auxiliary boiler.

The introduction of AE economizers in the mechanical propulsion configuration gives an additional $2.9 \%$ in overall efficiency and fuel OPEX improvement, compared to the standard options with ME economizers only. This also corresponds to a $7.3 \%$ improvement of the overall efficiency with a corresponding 5.9\% reduction in fuel OPEX, compared to the electric propulsion configuration. This technology is quite promising, with also lower capital cost, resulting in payback period of less than a year. The AE economizers can supply $39 \%$ of the steam demand.

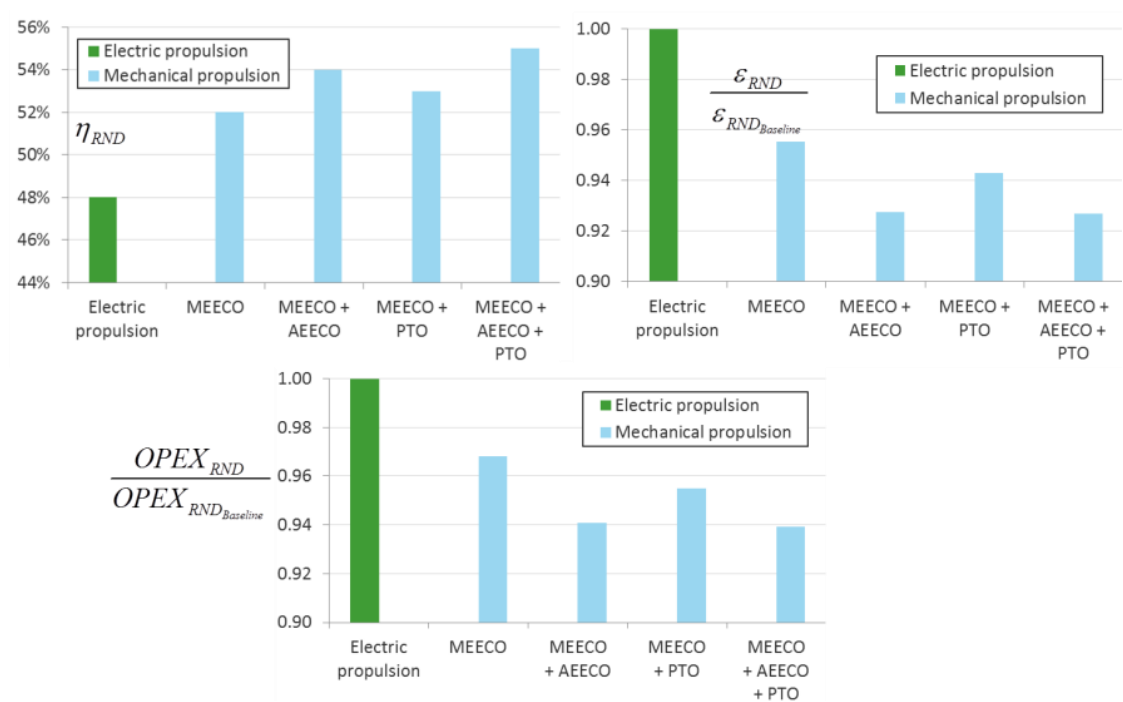

Figure. 11. Round-trip simulation results for the USA to Asia route for both electric and mechanical propulsion configurations and all energy recovery variants.

Table 3. Demand coverage ratios for the energy recovery variants for the USA to Asia route.

\begin{tabular}{|c|c|c|c|c|c|c|}
\hline & & \multirow{2}{*}{$\begin{array}{c}\text { Electric } \\
\text { propulsion }\end{array}$} & \multicolumn{4}{|c|}{ Mechanical propulsion variants } \\
\hline \multicolumn{2}{|c|}{ Coverage by: } & & MEECO & $\begin{array}{c}\text { MEECO + } \\
\text { AEECO }\end{array}$ & $\begin{array}{l}\text { MEECO + } \\
\text { PTO }\end{array}$ & $\begin{array}{c}\mathrm{MEECO} \mathrm{+} \\
\mathrm{AEECO} \mathrm{+} \mathrm{PTO}\end{array}$ \\
\hline \multirow{3}{*}{ Steam demand } & MEECO & 0.98 & 0.40 & 0.40 & 0.47 & 0.47 \\
\hline & AEECO & $\mathrm{n} / \mathrm{a}$ & $\mathrm{n} / \mathrm{a}$ & 0.39 & $\mathrm{n} / \mathrm{a}$ & 0.22 \\
\hline & $\mathrm{AB}$ & 0.02 & 0.60 & 0.21 & 0.53 & 0.31 \\
\hline \multirow{2}{*}{$\begin{array}{c}\text { Electricity } \\
\text { demand }\end{array}$} & PTO & $\mathrm{n} / \mathrm{a}$ & $\mathrm{n} / \mathrm{a}$ & $\mathrm{n} / \mathrm{a}$ & 0.41 & 0.41 \\
\hline & DFDE & 1.00 & 1.00 & 1.00 & 0.59 & 0.59 \\
\hline
\end{tabular}




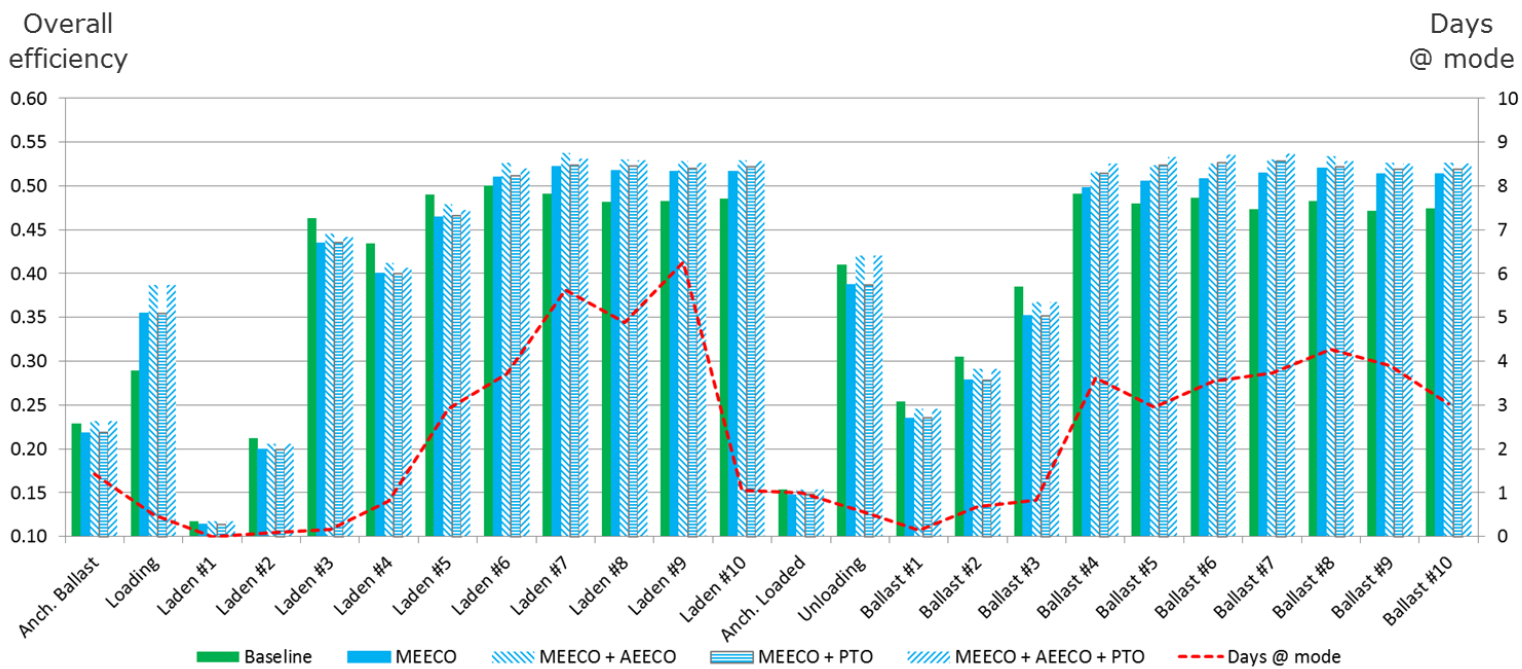

Figure. 12. Instantaneous overall efficiency per operating mode.

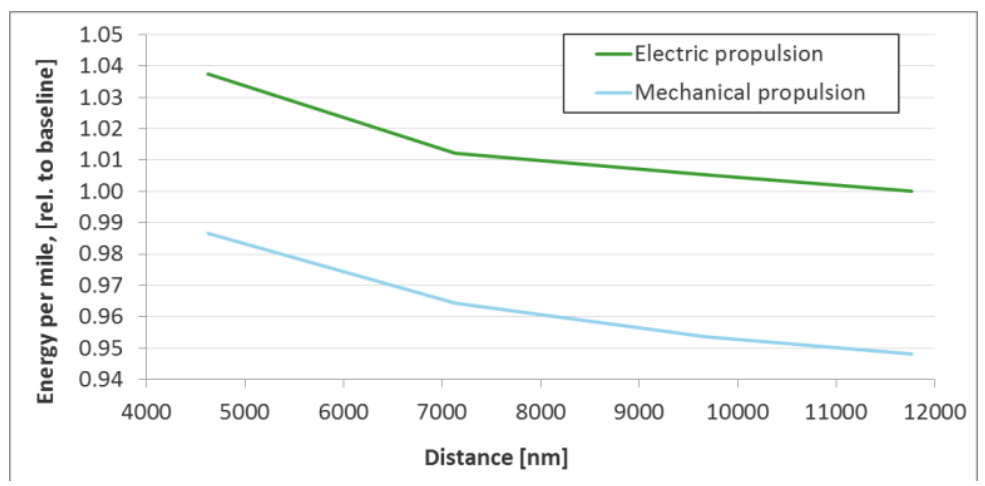

Figure. 13. Effect of sailing distance on energy consumption per nautical mile sailed.

The introduction of shaft generators (PTO) gives an additional $1 \%$ improvement in efficiency compared to the standard options with ME economizers only. This also corresponds to a $5.7 \%$ improvement of the overall efficiency with a corresponding $4.5 \%$ reduction in fuel OPEX, compared to the electric propulsion configuration. However, the capital cost for this solution is considerably higher, yielding a payback period of about 2 years. PTO can cover $41 \%$ of the electricity demand, with one auxiliary engine switched off.

The combination of both $\mathrm{AE}$ economizers and shaft generators yields the best results with an additional $2.6 \%$ improvement compared to the standard mechanical propulsion configuration. This also corresponds to a 7.3\% improvement of the overall efficiency with a corresponding $6.1 \%$ reduction in fuel OPEX, compared to the electric propulsion configuration. The payback period of this solution is approximately 1.7 years. The PTO again covers $41 \%$ of the electricity demand and the AE economizers cover $22 \%$ of the steam demand.

The simulation studies also yield results per operational mode. In Figure 12 the instantaneous overall efficiency (Eq. 8) of all configurations and energy efficiency variants is given per operating mode.

\subsection{Effect of Sailing Distance}

Finally, the effect of sailing distance has been considered. In Figure 13 the specific energy consumption per mile sailed are given in non-dimensional form as function of the route's sailing distance for the two configurations without energy recovery variants. The results indicate that longer routes are more energy efficient and cost effective. This is mainly due to the fact that the sailing operating modes are more efficient than the maneuvering, anchorage and port operations modes. Indicatively, a $12000 \mathrm{~nm}$ sailing route is 3\% more efficient than a $6000 \mathrm{~nm}$ route.

\section{Conclusions}

This work focused on the evaluation of different technology and configuration options for LNG carrier marine energy systems using process modelling techniques. LNG carriers feature complex and tightly integrated machinery systems, as well as time-varying operational profiles and trading patterns that require an integrated systems approach for their assessment and optimization at an early design stage.

The model-based assessment of the integrated machinery system presented in this paper proved to be particularly useful into identifying and quantifying the effect of the various design alternatives on efficiency and costs. The developed system models in our in-house process modelling framework DNVGL COSSMOS are generic, encompassing different machinery configurations and energy recovery technologies coupled with timevarying operating profiles.

The developed models were used in a number of model-based design and technology selection studies, identifying suitable gas compression sub-systems, BOG reliquefaction plant and combinations of energy recovery variants. An efficiency improvement of $8 \%$ was achieved in the presented case study, resulting in overall ship energy 
efficiency of more than $54 \%$. At the same time fuel savings of about $6 \%$ were identified, improving the overall costeffectiveness of the integrated system.

\section{Nomenclature}

$\dot{m}$ Mass flow rate, $\mathrm{kg} / \mathrm{s}$

$M \quad$ Mass, $\mathrm{kg} /$ round-trip

OPEX Operating expenses, USD

$\dot{Q} \quad$ Heat power, kW

$S_{\text {route }} \quad$ Route distance, $\mathrm{nm}$

$\dot{W} \quad$ Power, $\mathrm{kW}$

\section{Greek symbols}

$\Delta \tau \quad$ Duration, $\mathrm{h}$

$\varepsilon \quad$ Specific energy consumption, $\mathrm{kWh} / \mathrm{nm}$

$\eta \quad$ Efficiency

$\begin{array}{ll}\text { Subscripts and superscripts } \\ \text { cons } & \text { Consumption } \\ \text { D } & \text { Demand } \\ \text { el } & \text { Electricity } \\ \text { eng } & \text { Engines } \\ \text { exc } & \text { Excess } \\ \text { fcd } & \text { Forced } \\ \text { HFO } & \text { Heavy fuel oil } \\ \text { LHV } & \text { Lower Heating Value, } \mathrm{kJ} / \mathrm{kg} \\ \text { LNG } & \text { Liquefied Natural Gas } \\ \text { Nat } & \text { Natural } \\ \text { Nom } & \text { Nominal } \\ \text { PFO } & \text { Pilot fuel } \\ \text { PM } & \text { Propulsion motors } \\ \text { RLQ } & \text { Reliquefaction } \\ \text { RND } & \text { Round-trip } \\ \text { SG } & \text { Shaft generators } \\ \text { st } & \text { Steam }\end{array}$

\section{References}

[1] S. Wang, \& T. Notteboom, "Shipowners' structure and fleet distribution in the liquefied natural gas shipping market," Int. J. Shipping Transport Logistics, 6, 488512, 2014.

[2] M. Andreola, \& G. Tirelli, "Dual-fuel-electric LNG carrier machinery: when a concept becomes reality," Detail Wärtsilä Technical J., 2, 33-36, 2007.

[3] G. G. Dimopoulos, \& C. A. Frangopoulos, "A dynamic model for liquefied natural gas evaporation during marine transportation," Int. J.Thermodynamics, 11, 123131, 2008.

[4] G. G. Dimopoulos \& C. A. Frangopoulos, "Thermoeconomic simulation of marine energy systems for a Liquefied Natural Gas carrier," Int. J. Thermodynamics, 11, 195-201, 2008.

[5] G. G. Dimopoulos \& C. A. Frangopoulos, "Optimization of propulsion systems for modern LNG carriers considering multiple technology and design alternatives," 10th International Marine Design Conference, 2009.

[6] M. Wenninger \& S. Tolgos (2008). LNG Carrier Power (MAN Diesel Technical Paper), MAN, Ausburg, Germany.

[7] G. G. Dimopoulos, C. A. Georgopoulou, I. C. Stefanatos, A. S. Zymaris, \& N. M. P. Kakalis, "A general-purpose process modelling framework for marine energy systems," Energy Conversion and Management, 86, 325-339, 2014.

[8] PSE: Process Systems Enterprise, gPROMS [Online]. Available: www.psenterprise.com/gproms (accessed 1997-2009).

[9] J. R. Gómez, M. R. Gómez, R. F. Garcia, \& A. Catoira, "On board LNG reliquefaction technology: a comparative study," Polish Maritime Research, 21, 7788, 2013.

[10] R. Rao, \& V. Patel, "Thermodynamic optimization of cross flow plate-fin heat exchanger using a particle swarm optimization algorithm," Int. J. Thermal Sciences, 49(9), 1712-1721, 2010.

[11] Y. Shin, \& Y. P. Lee, "Design of a boil-off natural gas reliquefaction control system for LNG carriers," Applied Energy, 86, 37-44, 2009.

[12] MAN Diesel SE: Marine diesel engines Project Guides [Online]. Available : www.mandiesel.com (accessed 2011).

[13] Wartsila Corporation: Marine diesel engines Project Guides [Online]. Available: www.wartsila.com (accessed 2011).

[14] Dimopoulos, G. G. (2009). Synthesis, Design and Operation Optimization of Marine Energy Systems (Unpublished PhD Thesis), National Technical University of Athens, Athens, Greece. 